\title{
Nonequilibrium phase transitions in models of adsorption and desorption
}

\author{
R. Rajesh $*$ \\ Martin Fisher School of Physics, Brandeis University, Mailstop 057, Waltham, MA 02454-9110, USA
}

(Dated: November 7, 2018)

\begin{abstract}
The nonequilibrium phase transition in a system of diffusing, coagulating particles in the presence of a steady input and evaporation of particles is studied. The system undergoes a transition from a phase in which the average number of particles is finite to one in which it grows linearly in time. The exponents characterizing the mass distribution near the critical point are calculated in all dimensions.

PACS numbers: 05.70.Ln, 64.60.Ht, 68.55.-a
\end{abstract}

\section{INTRODUCTION}

There is a variety of physical phenomena in which the processes of diffusion, coagulation, adsorption and desorption play an important role. For example, submonolayer epitaxial thin film growth involves deposition of atoms onto a substrate and diffusion of these atoms leading to their aggregation into islands of increasing size [1]. A second example is river networks which have been modeled by aggregating masses in a steady influx of particles [2, 3]. Further examples include aerosols and clouds [4], colloids [5], and polymerization [6].

A simple lattice model that incorporates the above processes is the In-Out model 7, 8] in which diffusing point size particles on a lattice coagulate together on contact forming particles of larger mass. In addition unit mass is input uniformly at rate $q$ while unit mass evaporates from an existing mass at rate $p$. The competition between adsorption and desorption results in a nonequilibrium phase transition between a phase in which the average mass in the system is finite to one in which it increases linearly with time. A quantity that captures the features of the steady state is the mass distribution $P(m, t) . P(m, t)$ is the probability that a randomly chosen site has mass $m$ at time $t$. For fixed desorption rate $p$, the distribution $P(m, t)$ for large times was shown to change from an exponential distribution at small values of $q$ to a power law $m^{-\tau_{c}}$ at $q=q_{c}(p)$ and to a different power law $m^{-\tau}$ for $q>q_{c}(p)$. Near the transition point, $P\left(m, q-q_{c}, t\right)$ was seen to have the scaling form $P\left(m, q-q_{c}, t\right) \sim m^{-\tau_{c}} Y\left[m\left(q-q_{c}\right)^{\phi}, m t^{-\alpha}\right]$. The values of these exponents in high dimensions were calculated using a mean field approximation. In one dimension, they were determined using monte carlo simulations. The numerical values in one dimension were significantly different from the mean field results. In this paper, these exponents are calculated in all dimensions. The model is also extended to one in which particles diffuse with a mass dependent rate $m^{-\mu}$ with $\mu \geq 0$. The critical exponents for this more general model are also calculated.

Related models have been studied in the context of

*Electronic address: rrajesh@brandeis.edu nonequilibrium wetting [9, 10]. If the mass $m$ in the InOut model is identified with the height of a substrate, then the transition observed is qualitatively similar to nonequilibrium wetting transitions. In these models, the system undergoes a transition from a phase in which the interface is smooth to one in which it is rough. The exponents describing these transitions have been found to be related to some underlying contact process undergoing an absorbing to active transition. The In-Out model studied in this paper differs from these models by the lack of a surface tension term which tries to smoothen out the interface, and thus belongs to a different universality class.

The rest of the paper is organized as follows. In Sec. III the model is defined, the results obtained in Ref. [8] for the $\mu=0$ case are reviewed, and the results of this paper are summarized. In Sec. III a scaling relation is derived between the critical exponents. In Sec. IV] the phase in which the mean mass increases linearly with time is fully characterized. In Sec. $\mathbf{\nabla}$ exactly solvable limits of the model, namely $\mu=1$ case, zero dimensions and mean field solution, are discussed. In Sec. [VI the exponents for arbitrary $\mu$ are derived in all dimensions with the help of an assumption. The results are compared with monte carlo simulations in one dimension. In Sec. VII the results of the model are compared with results of related models. Section VIII contains a summary and concluding remarks. The appendices contain the details of the calculations.

\section{MODEL AND RESULTS}

\section{A. Definition}

For simplicity, we define the model on a one dimensional lattice with periodic boundary conditions; generalizations to higher dimensions is straight forward. Each site $i$ of the lattice has a non-negative integer mass variable $m_{i} \geq 0$. Given a certain configuration of masses at time $t$, the system evolves in an infinitesimal time $d t$ as follows. A site $i$ is chosen at random (with probability $d t$ ), and then the following events can occur. (i) Adsorption: with probability $q /(p+q+1)$, unit mass is adsorbed at site $i$; thus $m_{i} \rightarrow m_{i}+1$. (ii) Desorption: 
if the mass $m_{i}$ is greater than zero, then with probability $p /(p+q+1)$, unit mass is desorbed from site $i$; thus $m_{i} \rightarrow m_{i}-1$ provided $m_{i} \geq 0$. (iii) Diffusion and aggregation: if the mass $m_{i}$ is greater than zero, then with probability $m_{i}^{-\mu} /(p+q+1)$ the mass $m_{i}$ moves to a randomly chosen nearest neighbor. If the target site already happens to have some mass, then the total mass just adds up; thus $m_{i} \rightarrow 0$ and $m_{i \pm 1} \rightarrow m_{i \pm 1}+m_{i}$. The initial condition is chosen to be to be one in which all sites have mass zero. The model has three parameters, $p, q, \mu$.

\section{B. Review of results for $\mu=0$}

For $\mu=0$, when all particles diffuse at the same rate, the single site mass distribution at time $t, P(m, t)$ was determined in large dimensions using a mean field approximation and in one dimension using monte carlo simulations [8]. It was shown that when the adsorption rate $q$ was increased keeping the desorption rate $p$ fixed, the system undergoes a nonequilibrium phase transition across a critical line $q_{c}(p)$ from a phase in which $P(m)=\lim _{t \rightarrow \infty} P(m, t)$ has an exponential tail to one in which it has an algebraic tail for large mass; i.e,

$$
P(m) \sim \begin{cases}e^{-m / m^{*}} & \text { when } q<q_{c} \\ m^{-\tau_{c}} & \text { when } q=q_{c} \\ m^{-\tau} & \text { when } q>q_{c}\end{cases}
$$

where $m^{*}$ is a $q$ dependent cutoff, and $\tau$ and $\tau_{c}$ are exponents characterizing the power law decay. In addition, it was argued that as a function of the small deviation $\tilde{q}=q-q_{c}$, and large time $t, P(m, \tilde{q}, t)$ displays the scaling form

$$
P(m, \tilde{q}, t) \sim \frac{1}{m^{\tau_{c}}} Y\left(m \tilde{q}^{\phi}, \frac{m}{t^{\alpha}}\right),
$$

in terms of three unknown exponents $\phi, \alpha, \tau_{c}$, and the two variable scaling function $Y$. The the three phases will be called as the exponential phase $\left(q<q_{c}\right)$, the critical phase $\left(q=q_{c}\right)$ and the growing phase $\left(q>q_{c}\right)$.

Of interest are two more exponents. The mass at each site could be thought of as representing the height of an interface. In that case, two quantities of interest are the average velocity of the interface $F=d\langle m\rangle / d t$ and the fluctuations of the interface about its mean which would be dominated by $\left\langle m^{2}\right\rangle$, where $\langle\ldots\rangle$ denotes a spatial average. In the exponential phase, $\langle m\rangle$ is finite and hence $F=0$. In the growing phase, the interface has a finite velocity, and the velocity increases from zero as $F \sim \tilde{q}^{\theta}$, where $\theta$ is an exponent. At the critical point $\left\langle m^{2}\right\rangle \sim t^{\beta}$. Using the scaling form Eq. (2), it is straightforward to derive [8]

$$
\begin{aligned}
\theta & =\phi\left[1-\alpha\left(2-\tau_{c}\right)\right] / \alpha \\
\beta & =\alpha\left(3-\tau_{c}\right) .
\end{aligned}
$$

The model when $\mu=0$ was studied using a mean field approximation that ignored the spatial correlations between masses at different sites [8]. It was shown that $\phi=1, \alpha=2 / 3$ and $\tau_{c}=5 / 2$ when $\mu=0$. Correspondingly, $\theta=2$ and $\beta=1 / 3$. In one dimension, the exponents when $\mu=0$ were determined numerically to be $\tau_{c} \approx 1.83, \alpha \approx 0.61, \phi \approx 1.01, \theta \approx 1.47$, and $\beta \approx 0.71$.

\section{Summary of results}

In this paper, the In-Out model is studied for $\mu \geq 0$. Using scaling arguments, a relation is derived between the exponents $\alpha$ and $\tau_{c}$, thus reducing the number of unknown exponents from three to two. In particular, it is shown that

$$
\alpha\left(\mu d+2 \tau_{c}-2\right)=d, \quad d \leq 2 .
$$

The exponent $\tau_{c}$ in $d \leq 2$ is shown to be

$$
\tau_{c}= \begin{cases}\frac{d^{2}+6 d+4}{2(d+2)} & \mu=0, \\ \frac{(3-\mu) d+2}{d+2} & 0<\mu<2 .\end{cases}
$$

The exponent $\alpha$ in $d \leq 2$ is shown to be

$$
\alpha= \begin{cases}\frac{d+2}{d+4} & \mu=0, \\ \frac{d+2}{\mu d+4} & 0<\mu<2 .\end{cases}
$$

The exponent $\phi$ in $d \leq 2$ is calculated for $\mu=0$ and $\mu=1$ :

$$
\phi= \begin{cases}1 & \mu=0 \\ \frac{d+2}{2} & \mu=1\end{cases}
$$

In the growing phase, the exponent $\tau$ characterizing the power law decay of the mass distribution is shown to be

$$
\tau=\frac{(2-\mu) d+2}{d+2} .
$$

In one dimension, when $\mu=0$, the exponents reduce to $\tau_{c}=11 / 6, \alpha=3 / 5$ and $\phi=1$. This is in very good agreement with the numerical results seen in Ref. [8] (see Sec. IIB). In dimensions greater than two, the exponents take on their mean field value, obtained by setting $d$ to 2 in the above equations.

\section{SCALING RELATION BETWEEN $\alpha$ AND $\tau_{c}$}

In this section, a relation between $\alpha$ and $\tau_{c}$ is obtained from scaling arguments for all $\mu$. The dependence of the largest mass in the system $M_{t}$ on $t$ can be obtained by the catchment area argument as follows. Due to diffusion, the mass $M_{t}$ would sweep out an area $L_{t}^{d}$ in time $t$, where $L_{t}$ is the typical length scale in the system. In addition to the mass contained in this area, $M_{t}$ also increases due to the average flux $F=d\langle m\rangle / d t$. Thus,

$$
M_{t} \sim L_{t}^{d} F t .
$$


The typical length $L_{t}$ arises from diffusion: $L_{t} \sim(D t)^{1 / 2}$. Substituting $M_{t}^{-\mu}$ for the diffusion rate $D$, and using $F \sim t^{\alpha\left(2-\tau_{c}\right)-1}$ from the scaling relation Eq. (2), we obtain $M_{t} \sim t^{\left(d+2 \alpha\left(2-\tau_{c}\right)\right) /(2+\mu d)}$. But, by definition (see Eq. (2)) $M_{t} \sim t^{\alpha}$. Equating the exponents, we obtain

$$
\alpha\left(\mu d+2 \tau_{c}-2\right)=d .
$$

Thus, the number of unknown exponents reduces from three to two. The above scaling arguments are valid only when $L_{t}$ increases as a positive power of $t$. This restriction translates to the condition $\alpha \mu<1$.

\section{THE GROWING PHASE $\left(q>q_{c}\right)$}

In this section, the behavior of $P(m)$ in the growing phase $q>q_{c}$ is discussed. The exponent $\tau$ (as defined in Eq. (10) is expected to be independent of the precise values of $q$ and $p$ as long as we are above the critical threshold [8]. To obtain $\tau$, the convenient limit $p=0$ and $q$ arbitrary may be studied. Different aspects of this limiting case have been studied in the context of river networks, self organized criticality and epitaxial growth 11, 12, 13, 14, 15, 16. We give a short derivation of the exponent $\tau$. In this limiting case of only adsorption, it is known that $P(m, t)$ has the scaling form

$$
P(m, t) \sim m^{-\tau} f\left(\frac{m}{t^{\delta}}\right),
$$

where the scaling function $f(x)$ tends to a constant (for $\mu<1$ ) for small values of $x$ and decays exponentially for large values of $x$. Since there is a constant influx $F$ of particles, $\langle m\rangle=F t$. Therefore $\delta(2-\tau)=1$. To obtain a second relation between the exponents, note that Eq. (11) is valid when $\tau_{c}$ is replaced by $\tau$ and $\alpha$ by $\delta$. Solving these two exponent equalities, one obtains

$$
\begin{aligned}
& \tau=\frac{(2-\mu) d+2}{d+2}, \\
& \delta=\frac{d+2}{\mu d+2} .
\end{aligned}
$$

Equation (13) is valid when $\mu<1$ and $d \leq 2$. For $d>2$, the mean field results are correct. The $\mu=0$, results were obtained earlier in 11, 12, 13. For $\mu>0$, the one and two dimensional results were obtained earlier 14, 15]. The dependence of $P(m, t)$ on the flux $F$ can now be incorporated into Eq. (12) by simple dimensional arguments:

$$
P(m, t) \sim \frac{F^{\frac{d}{d+2}}}{m^{\tau}} f\left(\frac{m}{\left(F^{\frac{2}{d+2}} t\right)^{\delta}}\right),
$$

where $\tau$ and $\delta$ are as in Eq. (13).

The two variable scaling function $Y(x, y)$ in Eq. (2) should be such that when $x \gg 1$ (or $m \tilde{q}^{\phi} \gg 1$ ), it reduces to the one variable scaling function $f$ in Eq. (14). This implies that $Y(x, y) \sim x^{\tau_{c}-\tau} f\left(y / x^{1-\alpha / \delta}\right)$ when $x \gg 1$. Thus

$$
P(m, t) \sim \frac{\tilde{q}^{\phi\left(\tau_{c}-\tau\right)}}{m^{\tau}} f\left(\frac{m}{\left(\tilde{q}^{\gamma} t\right)^{\delta}}\right),
$$

where $\gamma$ is a crossover exponent. To make a comparison with Eq. (14), one has to make the identification $F \sim \tilde{q}^{\theta}$. Using Eqs. (3), (11) and (13), it is easy to show that $\tilde{q}^{\phi\left(\tau_{c}-\tau\right)} \sim F^{d /(d+2)}$. Also,

$$
\gamma=2 \theta /(d+2) .
$$

\section{SOLVABLE LIMITS}

In this section, we examine limiting cases of the model which are analytically tractable. For the sake of continuity of argument, the details of the calculation are deferred to the appendices.

\section{A. Solution for $\mu=1$}

The special case when a mass $m$ diffuses as $m^{-1}$ can be solved by examining the time evolution of the two point correlations. When $\mu=1$, certain simplifications occur. We refer to Appendix $\mathrm{A}$ for details. It is shown that the critical $q_{c}$ at which the mean mass increases with time is

$$
q_{c}=d p^{2} g(p),
$$

where

$$
g(p)=\int_{0}^{2 \pi} \frac{d k_{1}}{2 \pi} \ldots \int_{0}^{2 \pi} \frac{d k_{d}}{2 \pi} \frac{1}{(1+p) d-\sum_{i=1}^{d} \cos \left(k_{i}\right)} .
$$

The exponents for $\mu=1$ is shown to be (see Appendix

$$
\begin{aligned}
\tau_{c} & =\frac{2 d+2}{d+2}, \\
\alpha & =\frac{d+2}{d+4}, \\
\phi & =\frac{d+2}{2} .
\end{aligned}
$$

Solving for $\theta$ from Eq. (3), we obtain $\theta=(d+2) / 2$.

\section{B. Mean field theory}

The exponents may be computed in large dimensions by a mean field analysis. This approximation involves ignoring the correlations between masses at two different sites. The details of the calculation are presented in Appendix [B] The results are

$$
\tau_{c}= \begin{cases}\frac{5}{2} & \mu=0 \\ 2-\frac{\mu}{2} & 0<\mu<2 .\end{cases}
$$


The exponent $\alpha$ in the mean field equals

$$
\alpha= \begin{cases}\frac{2}{3} & \mu=0 \\ \frac{2}{2+\mu} & 0<\mu<2 .\end{cases}
$$

The exponent $\phi$ could be computed only for $\mu=0$ and $\mu=1$ :

$$
\phi= \begin{cases}1 & \mu=0, \\ 2 & \mu=1 .\end{cases}
$$

Using Eqs. (3) and (16), we obtain

$$
\begin{array}{ll}
\theta=2 & \mu=0,1, \\
\gamma=1 & \mu=0,1 .
\end{array}
$$

Comparison with the exact solution for $\mu=1$ or calculating the dimension $d$ when the mean field exponents satisfy the scaling relation Eq. (11) shows that the upper critical dimension of the system is 2 .

\section{Solution for $d=0$}

In zero dimensions, the problem may be solved for in a straightforward manner (see Appendix C). The exponents are independent of $\mu$, since there is no diffusion. In this case,

$$
\begin{aligned}
\tau_{c} & =1, \\
\alpha & =\frac{1}{2}, \\
\phi & =1,
\end{aligned}
$$

for all values of $\mu$. Using Eqs. (3) and (16), we obtain

$$
\begin{aligned}
& \theta=1, \\
& \gamma=1 .
\end{aligned}
$$

\section{EXPONENTS FOR ARBITRARY $\mu$ AND $d$}

The question remains as to what the values of the exponents are in arbitrary dimensions. They can be determined with the help of an assumption. We make the assumption that the critical exponents for a given $\mu$ are a monotonic function of dimension $d$. This assumption is reasonable as known exponents for most systems at their critical point (for example, the Ising model) have this property.

Consider first the exponents when $\mu=0$. Notice that the exponent $\phi$ for $\mu=0$ takes on the same value in the mean field or $d=2$ (see Eq. (24)) as well as in $d=0$ (see Eq. (29)). Assuming that $\phi(d)$ should be monotonic in $d$, we obtain

$$
\phi=1, \quad \mu=0 .
$$

Consider now the exponent $\gamma$ (as defined in Eq. 15). It takes the value 1 in $d=0$ (see Eq. 31) and in the mean

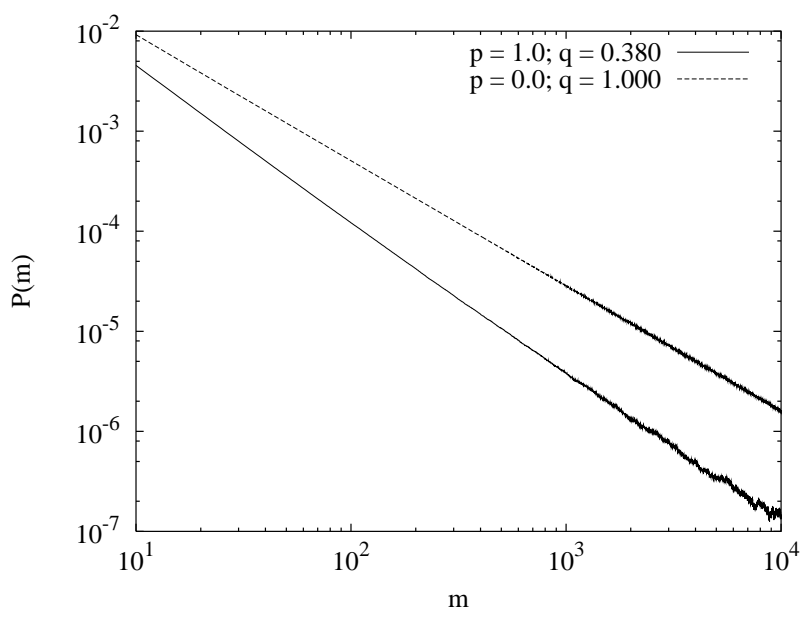

FIG. 1: The variation of $P(m)$ with $m$ is shown at the critical point (bottom curve) and in the growing phase (top curve). The results are for $\mu=0.25$. A best fit for the curves give exponent values $\tau_{c}=1.58 \pm 0.04$ and $\tau=1.25 \pm 0.01$.

field limit (see Eq. 26). Hence, $\gamma=1$ in all dimensions or

$$
\theta=\frac{d+2}{2}, \quad \mu=0 .
$$

Numerical simulations of the $\mu=0$ model in one dimension had $\phi \approx 1.01$ and $\theta \approx 1.47[8]$ consistent with the above results. Knowing $\theta$, the exponents $\tau_{c}$ and $\alpha$ can be solved for from Eqs. (31) and (11) to yield

$$
\begin{aligned}
& \tau_{c}=\frac{d^{2}+6 d+4}{2(d+2)}, \quad \mu=0, \\
& \alpha=\frac{d+2}{d+4}, \quad \mu=0 .
\end{aligned}
$$

Specializing to $d=1$, the exponents reduce to $\tau_{c}=11 / 6$ and $\alpha=3 / 5$. Again, these values are very close to the numerical values of 1.83 and 0.61 obtained in Ref. 8].

When $\mu>0$, we can calculate the exponent $\tau_{c}$ and $\alpha$ as follows. Consider the exponent $\beta$ defined by $\left\langle m^{2}\right\rangle \sim t^{\beta}$ at the critical point. Clearly $\beta=\alpha\left(3-\tau_{c}\right)$. Note that when $\mu>0, \beta=1$ in the mean field analysis as well as in zero dimensions. Thus, using the argument of monotonicity of exponents, we obtain

$$
\alpha\left(3-\tau_{c}\right)=1, \quad \mu>0 .
$$

Solving Eqs. (11) and (36), we obtain

$$
\begin{aligned}
\tau_{c} & =\frac{(3-\mu) d+2}{d+2}, \quad 0<\mu<2, \\
\alpha & =\frac{d+2}{\mu d+4}, \quad 0<\mu<2 .
\end{aligned}
$$

When $\mu=1$ or when $d=2$, the results match the exact results derived in Sec. IV] The exponent $\phi$ for $\mu>0$ is 


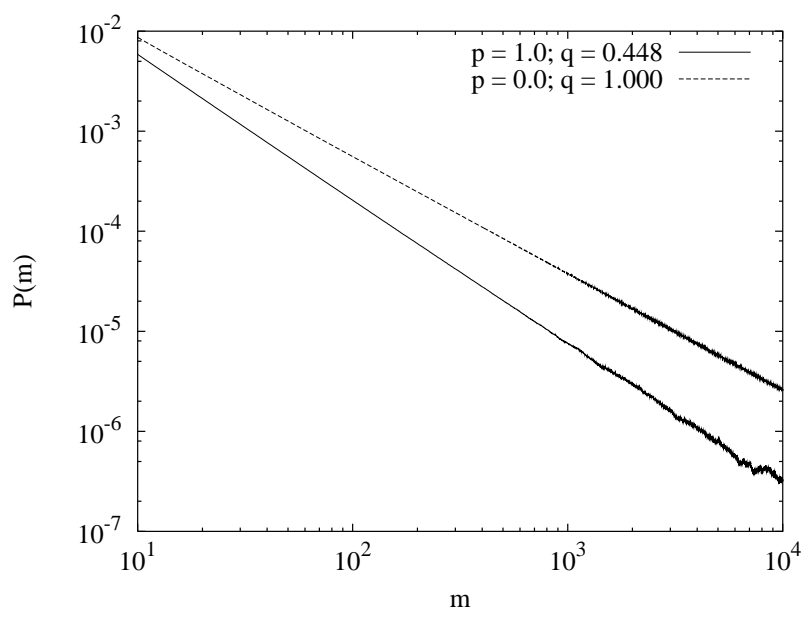

FIG. 2: The variation of $P(m)$ with $m$ is shown at the critical point (bottom curve) and in the growing phase (top curve). The results are for $\mu=0.50$. A best fit for the curves give exponent values $\tau_{c}=1.47 \pm 0.04$ and $\tau=1.17 \pm 0.01$.

still undetermined, and there seems to be no easy way to calculate it.

The analytical results are now compared with results from monte carlo simulations in one dimension. When $\mu=0$, simulations were done in Ref. [8]. As pointed out earlier in this section, the numerical values of the exponents are in close agreement with that obtained in this paper. We therefore concentrate on non-zero values of $\mu$. The exponent that is determined numerically is $\tau_{c}$ for $\mu=0.25$ and $\mu=0.5$. The simulations were done on a one dimensional lattice of size 1000 with periodic boundary conditions. $P(m)$ was obtained by averaging over $10^{8}$ realizations.

In Fig. 11 the results for $\mu=0.25$ is shown. $P(m)$ is measured for $q=q_{c} \approx 0.380$ when $p=1.0$, and for the growing phase in which $p$ is set to zero. The critical $q_{c}$ was fixed to be that value of $q$ at which the distribution changed from an exponential to a power law. A best fit gives $\tau_{c}=1.58 \pm 0.04$ and $\tau=1.25 \pm 0.01$. These should be compared with the analytical results $\tau_{c}=1.583 \ldots$ and $\tau=1.25$.

Fig. 2] is as in Fig. 11 except that $\mu=0.5$ and $q_{c} \approx 0.448$. A best fit gives $\tau_{c}=1.47 \pm 0.04$ and $\tau=1.17 \pm 0.01$. These should be compared with the analytical results $\tau_{c}=1.5 \ldots$ and $\tau=1.166 \ldots$.

\section{CONNECTION TO RELATED MODELS}

In this section, similarities between the In-Out model and other related models of aggregation are discussed. A model that closely resembles the model studied in this paper is the charge model with adsorption [17, 18, 19]. In this model, there is no longer the restriction that the masses have to be non-negative. Also, +1 and -1 masses are input at the same rate. In the limit of large time,
$P(m)$ for this model is a power law $P(m) \sim|m|^{-5 / 3}$ for $|m| \gg 1$ in one dimension. This model could be expected to have the same behavior as the In-Out model at the critical point, since the growth velocity is zero. However, the exponent for the charge model is different from the value $11 / 6$ obtained for the In-Out model, showing that the restriction of non-negative masses is relevant. We now ask whether it is relevant when $\mu>0$.

Since the charge model was not studied earlier for $\mu>0$, we now give a short derivation of the power law exponent using scaling arguments. It was shown based on very general arguments that for the charge model, irrespective of the diffusion rates that 19]

$$
\left\langle m^{2}\right\rangle \sim t, \quad t \gg 1
$$

Assuming scaling for $P(m, t)$ as in Eq. (12) and the scaling relation Eq. (11), it is easy to see that $P(m) \sim m^{-\tau_{c h}}$, where

$$
\tau_{c h}=\frac{(3-\mu) d+2}{d+2}, \quad 0<\mu<2
$$

But this is the same as $\tau_{c}$ obtained for the In-Out model (see Eq. (37). Thus, the charge model and the In-Out model appears to have the same behavior when $\mu>0$.

A reason for this could be the following. When $\mu=0$, there is a chance that large positive masses get neutralized by large negative masses in the charge model. This process is totally absent in the In-Out model, resulting in the exponents being different. When $\mu>0$, large positive and negative charges get immobilized and their collision becomes infrequent. Hence, one could ignore this process in the charge model and hence the two models become qualitatively similar. A pitfall of this argument is that it predicts that $\tau_{c}$ for $\mu=0$ should be less than $\tau_{c h}$, contrary to what is seen. Thus, the exact connection between these two models remains unclear.

Another model which is related to the In-Out model is a model of coagulation with fragmentation 7, 20, 21]. In this model, the desorption at a site is accompanied by adsorption at the neighboring site, thus conserving mass locally. In this model, there is a phase transition from a phase in which $P(m)$ is exponentially distributed to one in which it is a power law, accompanied by a infinite aggregate which accommodates a finite fraction of the total mass. When the diffusion constant is massdependent, it was shown that 21], the exponent $\tau_{c}$ in the mean field limit is exactly the same as that of the In-Out model in the mean field limit. However, in dimensions lower than the upper critical dimension, the exponents in the model with mass conservation remains equal to the mean field value, unlike the In-Out model.

\section{SUMMARY AND CONCLUSIONS}

To summarize, the exponents characterizing the phase transition from a phase with finite mean height to one 
in which it grows linearly with time in the In-Out model were calculated. The model was extended to one in which particles diffuse with a mass dependent rate $D(m) \propto$ $m^{-\mu}$. The exponents were shown to have a discontinues jump at $\mu=0$. The exponents are unrelated to previously studied universality classes of nonequilibrium phase transitions.

There are several questions that remain unanswered. Other models which show a wetting transition as seen in the In-Out model have exponents which can be expressed in terms of exponents of absorbing phase transitions [9, 10]. Here, there seems to be no apparent connection to any underlying absorbing phase transition. It would be interesting to find connections to other models of nonequilibrium phase transitions.

The calculation of exponents in this paper for arbitrary $\mu$ relied on the assumption that the exponents are monotonic with dimension. While simulations do support the results that are obtained, it is important to have a more rigorous derivation of the exponents without making this assumption. Also, one would expect logarithmic corrections to the power laws in two dimensions. These have been ignored in this paper. A calculation of these corrections would be of interest.

A connection to the charge model was pointed out in Sec. VII The models seem to be similar for $\mu>0$, while different for $\mu=0$. The precise connection between the two would be worth exploring since the charge model is analytically more tractable. Finally, the discontinuity of exponents at $\mu=0$ remains a puzzle.

\section{APPENDIX A: EXACT SOLUTION FOR $\mu=1$}

In this appendix, we derive the exponents for $\mu=1$ in arbitrary dimensions. We do so by examining the two point correlations in the system in the steady state. To fix notation, let $\mathbf{x}^{\prime}$ denote one of the $2 d$ nearest neighbors of the site $\mathbf{x}$. Let $\eta\left(\mathbf{x}, \mathbf{x}^{\prime}, t\right)$ be the mass transferred from site $\mathbf{x}$ to $\mathrm{x}^{\prime}$ at time $t$ in a time interval $\Delta t$. From the definition of the model, it follows that

$$
\eta\left(\mathbf{x}, \mathbf{x}^{\prime}, t\right)= \begin{cases}m_{\mathbf{x}} & \text { with prob. } \frac{1}{2 d} \frac{\Delta t}{m_{\mathbf{x}}^{\mu}} \\ 0 & \text { otherwise. }\end{cases}
$$

To order $\Delta t$, the only nonzero two point correlation in the noise is

$$
\left\langle\eta\left(\mathbf{x}, \mathbf{x}^{\prime}\right)^{2}\right\rangle=\frac{m_{\mathbf{x}}^{2-\mu} \Delta t}{2 d} .
$$

Let $I(\mathbf{x}, t)$ be the mass transferred due to adsorption and desorption from the site $\mathbf{x}$ at time $t$ in an infinitesimal time $\Delta t$. Then,

$$
I(\mathbf{x}, t)= \begin{cases}1 & \text { with prob } q \Delta t, \\ -1+\delta_{m_{\mathbf{x}}, 0} & \text { with prob } p \Delta t, \\ 0 & \text { otherwise. }\end{cases}
$$

To order $\Delta t$, the only nonzero two point correlation in the input $I$ is

$$
\left\langle I(\mathbf{x}, t)^{2}\right\rangle=(q+p s) \Delta t
$$

where $s=\sum_{m=1} P(m)$ is the occupation probability.

The mass $m_{\mathbf{x}}(t)$ at lattice site $\mathbf{x}$ at time $t$ evolves as

$$
\begin{aligned}
m_{\mathbf{x}}(t+\Delta t)= & m_{\mathbf{x}}(t)-\sum_{\mathbf{x}^{\prime}} \eta\left(\mathbf{x}, \mathbf{x}^{\prime}, t\right)+\sum_{\mathbf{x}^{\prime}} \eta\left(\mathbf{x}^{\prime}, \mathbf{x}, t\right) \\
& +I(\mathbf{x}, t) .
\end{aligned}
$$

To obtain the two point correlations, we multiply $m_{\mathbf{x}}(t+$ $\Delta t)$ by $m_{\mathbf{0}}(t+\Delta t)$ and take averages over the possible stochastic moves. Using Eqs. A1 - A5), we obtain

$$
\begin{aligned}
\frac{d C(\mathbf{x})}{d t}= & -2 C_{\mu}(\mathbf{x})+\frac{1}{d} \sum_{j=1}^{d} \sum_{k= \pm 1} C_{\mu}\left(x_{1}, \ldots, x_{j}+k, \ldots x_{d}\right)+2(q-p) \rho+2 p D(\mathbf{x}) \\
& +\delta_{\mathbf{x}, 0}(q+p s)+\left\langle m^{2-\mu}\right\rangle\left(2 \delta_{\mathbf{x}, \mathbf{0}}-\frac{1}{d} \sum_{j=1}^{d} \sum_{k= \pm 1} \delta_{x_{1}, 0} \ldots \delta_{x_{j}+k, 0} \ldots \delta_{x_{d}, 0}\right\rangle
\end{aligned}
$$

where $C(\mathbf{x})=\left\langle m_{\mathbf{x}} m_{\mathbf{0}}\right\rangle, C_{\mu}(\mathbf{x})=\left\langle m_{\mathbf{x}} m_{\mathbf{0}}^{1-\mu}\right\rangle, \rho=\langle m\rangle$ and $D(\mathbf{x})=\left\langle m_{\mathbf{x}} \delta_{m_{\mathbf{0}}, 0}\right\rangle$.

Consider Eq. (A6) in the steady state when the time derivative is set to zero. For arbitrary values of $\mu$, the right hand side of Eq. A6 involves three unknowns: $C_{\mu}(\mathbf{x}), D(\mathbf{x})$ and $\rho$. However, when $\mu=1$, a simplification occurs because

$$
C_{1}(\mathbf{x})=\rho-D(\mathbf{x})
$$

thus reducing the number of unknowns to two. Define

$$
F(\mathbf{k})=\sum_{\mathbf{x}}[D(\mathbf{x})-\rho(1-s)] e^{i \mathbf{k} \cdot \mathbf{x}}
$$

Solving for $F(\mathbf{k})$ from Eq. A6 , we obtain

$$
F(\mathbf{k})=-\rho+\frac{q d-p \rho d}{h(\mathbf{k})}
$$


where

$$
h(\mathbf{k})=\sum_{i=1}^{d} \cos \left(k_{i}\right)-(1+p) d .
$$

To obtain $\rho$, we use the fact that the constant term in $F(\mathbf{k})$ equals $-\rho(1-s)$. Then

$$
\rho=\frac{d p q g(p)}{d p^{2} g(p)-q},
$$

where

$$
g(p)=\int_{0}^{2 \pi} \frac{d k_{1}}{2 \pi} \ldots \int_{0}^{2 \pi} \frac{d k_{d}}{2 \pi} \frac{1}{(1+p) d-\sum_{i=1}^{d} \cos \left(k_{i}\right)} .
$$

Thus, the mean density diverges as $\left(q-q_{c}\right)^{-1}$, where

$$
q_{c}=d p^{2} g(p) .
$$

Specializing the result to one and two dimensions,

$$
\begin{aligned}
q_{c}^{1 D} & =\frac{p^{3 / 2}}{\sqrt{p+2}}, \\
q_{c}^{2 D} & =\frac{2 p^{2}}{\pi(1+p)} K\left[\frac{1}{1+p}\right],
\end{aligned}
$$

where $K$ is the complete elliptic integral of the first kind.

The form of the divergence of the density as $q$ approaches $q_{c}$, namely $\langle m\rangle \sim \tilde{q}^{-1}$ means that

$$
\phi\left(2-\tau_{c}\right)=1, \quad \text { for } \mu=1,
$$

in all dimensions. To obtain one more relation between the exponents, we consider $\frac{d\left\langle m^{2}\right\rangle}{d t}$ for large times at the critical point. Firstly, we need to invert Eq. A99 to obtain $D(\mathbf{1})$ where $\mathbf{1}$ denotes the site $(1,0,0, \ldots)$. Inverting, we obtain

$$
D(\mathbf{1})=\frac{\rho(p-q)}{q}+(q-p \rho)[1-d(1+p) g(p)] .
$$

We now make the assumption that the leading time dependence to $D(\mathbf{1})$ is obtained by restoring the time dependence of $\rho$. Then, when $\mathbf{x}=\mathbf{0}$, Eq. (A6) reduces to

$$
\frac{d\left\langle m^{2}\right\rangle}{d t} \approx 2 \rho(t) \frac{(1+p)\left(q-q_{c}\right)}{p}+2 q d(1+p) g(p) .
$$

If we now take the limit $q \rightarrow q_{c}$ before $t \rightarrow \infty$, then we obtain that $\left\langle m^{2}\right\rangle \sim t$. Thus

$$
\alpha\left(3-\tau_{c}\right)=1, \quad \text { for } \mu=1,
$$

in all dimensions. Solving for the exponents $\tau_{c}, \alpha$ and $\phi$ from Eqs. (11), A15 and (A18), we obtain

$$
\begin{aligned}
\tau_{c} & =\frac{2 d+2}{d+2}, \\
\alpha & =\frac{d+2}{d+4}, \\
\phi & =\frac{d+2}{2} .
\end{aligned}
$$

Correspondingly $\theta=(d+2) / 2$ and $\beta=1$.

\section{APPENDIX B: MEAN FIELD SOLUTION}

In this appendix, we derive the exponents for $\mu \geq 0$ using a mean field approximation. This approximation involves ignoring correlations between the masses, i.e., replacing joint probability distribution functions by product of single point distributions. Then, the master equation for the temporal evolution of $P(m, t)$ is

$$
\begin{aligned}
\frac{d P(m)}{d t} & =-\left(m^{-\mu}+s^{\prime}+p+q\right) P(m)+q P(m-1) \\
& +p P(m+1)+\sum_{m^{\prime}=1}^{m} \frac{P\left(m^{\prime}\right) P\left(m-m^{\prime}\right)}{m^{\prime \mu}} \\
\frac{d P(0)}{d t} & =s s^{\prime}-q+q s+p P(1),
\end{aligned}
$$

where $s=\sum_{m=1} P(m)$ and $s^{\prime}=\sum_{m=1} m^{-\mu} P(m)$. The different terms enumerate the number of ways the mass at a certain site can change. Then it follows that

$$
\begin{aligned}
\frac{d\left\langle m^{n}\right\rangle}{d t} & =\sum_{k=1}^{n-1}\left(\begin{array}{l}
n \\
k
\end{array}\right)\left\langle m^{k}\right\rangle\left\langle m^{n-k-\mu}\right\rangle+q+(-1)^{n} p s \\
& +\sum_{k=1}^{n-1}\left(\begin{array}{l}
n \\
k
\end{array}\right)\left[q+(-1)^{k} p\right]\left\langle m^{n-k}\right\rangle .
\end{aligned}
$$

Consider first the steady state solution of Eq. (B3) when the time derivatives may be set to zero. Then, putting $n=1$, and solving for the occupation probability $s$, we obtain

$$
s=\frac{q}{p} .
$$

The mean field equations take on a simpler form for the cases $\mu=0$ and $\mu=1$, and hence we solve them separately from the arbitrary $\mu$ case. Though all the mean field exponents for $\mu=0$ were derived in Ref. [8] using the generating function method, they will be rederived here using a different method which will be simpler to generalize to the $\mu \geq 0$ case.

$$
\text { 1. } \mu=0
$$

On choosing $n=2$ in Eq. (B3) and taking the steady state limit, a quadratic equation for $\langle m\rangle$ is obtained which can be solved to yield

$$
\langle m\rangle=\frac{p-q-\sqrt{(p-q)^{2}-4 q}}{2}
$$

The expression for $\langle m\rangle$ becomes invalid when expression under the square root sign becomes negative, thus fixing $q_{c}$. Solving, we obtain

$$
q_{c}=p+2-2 \sqrt{1+p},
$$


where the sign is chosen by the condition that $q_{c}=0$ at $p=0$. Consider now the equation corresponding to $n=3$ in Eq. (B33). Solving for $\left\langle m^{2}\right\rangle$, we obtain

$$
\left\langle m^{2}\right\rangle=\frac{(p+q)\langle m\rangle}{p-q-2\langle m\rangle} .
$$

But the denominator tends to zero as $\sqrt{\tilde{q}}$, where $\tilde{q}=$ $q_{c}-q$. Therefore, near the transition point $\left\langle m^{2}\right\rangle$ diverges as

$$
\left\langle m^{2}\right\rangle \sim \frac{1}{\tilde{q}^{1 / 2}} .
$$

Consider now the equation corresponding to $n=4$ in Eq. (B3). Solving for $\left\langle m^{3}\right\rangle$, we obtain

$$
\begin{aligned}
\left\langle m^{3}\right\rangle & =\frac{3\left\langle m^{2}\right\rangle^{2}+3(q+p)\left\langle m^{2}\right\rangle+2(q-p)\langle m\rangle+q}{2(p-q-2\langle m\rangle)}(\mathrm{B} 9) \\
& \sim \frac{1}{\tilde{q}^{3 / 2}}, \quad \tilde{q} \rightarrow 0 .
\end{aligned}
$$

Knowing the behavior of $\left\langle m^{2}\right\rangle$ and $\left\langle m^{3}\right\rangle$ near the transition point, we immediately obtain

$$
\begin{aligned}
& \phi\left(3-\tau_{c}\right)=\frac{1}{2}, \\
& \phi\left(3-\tau_{c}\right)=\frac{3}{2},
\end{aligned}
$$

which can be solved to give

$$
\begin{aligned}
\tau_{c} & =5 / 2, \\
\phi & =1 .
\end{aligned}
$$

To calculate $\phi$ and $\tau$ we had first taken the limit $t \rightarrow \infty$ followed by the limit $\tilde{q} \rightarrow 0$. In order to calculate $\alpha$, we need to take the limits in the opposite order, namely $\tilde{q} \rightarrow 0$ followed by $t \rightarrow \infty$. We first note that in this limit $s=q / p$ and $\langle m\rangle=(p-q) / 2$. Then choosing $n=3$ in Eq. B3] we obtain

$$
\frac{d\left\langle m^{3}\right\rangle}{d t}=\frac{3\left(p^{2}-q_{c}^{2}\right)}{2} .
$$

Thus,

$$
\alpha\left(4-\tau_{c}\right)=1 .
$$

Substituting for $\tau_{c}$, we obtain

$$
\alpha=\frac{2}{3} .
$$

Thus, in the mean field limit, the scaling function takes on the form

$$
P(m, \tilde{q}, t) \sim \frac{1}{m^{5 / 2}} Y\left(m \tilde{q}, \frac{m}{t^{2 / 3}}\right), \quad \mu=0 .
$$

$$
\text { 2. } \mu=1
$$

We start again with Eqs. (B3) and (B4). For $\mu=$ 1, Eq. (B3) simplifies because $\left\langle m^{n-k-\mu}\right\rangle$ reduces to an integer moment of $m$. Choosing $n=2$ in Eq. (B33) in the steady state, we obtain

$$
\langle m\rangle=\frac{p q}{p^{2}-p q-q} .
$$

The mean mass $\langle m\rangle$ diverges when $q=q_{c}$, where

$$
q_{c}=\frac{p^{2}}{1+p} .
$$

Choosing $n=3$ in Eq. (B33) in the steady state, we obtain

$$
\left\langle m^{2}\right\rangle=p \frac{\langle m\rangle^{2}+(q+p)\langle m\rangle}{p^{2}-p q-q} .
$$

Equations (B19) and B21] imply that $\langle m\rangle \sim \tilde{q}^{-1}$ and $\left\langle m^{2}\right\rangle \sim \tilde{q}^{-3}$ when $\tilde{q} \rightarrow 0$. Thus,

$$
\begin{aligned}
& \phi\left(2-\tau_{c}\right)=1 \\
& \phi\left(3-\tau_{c}\right)=3 .
\end{aligned}
$$

Solving, we obtain

$$
\begin{aligned}
\tau_{c} & =\frac{3}{2}, \\
\phi & =2 .
\end{aligned}
$$

In order to calculate $\alpha$, we need to set $q=q_{c}$ and take the large time limit. Then choosing $n=3$ in Eq. B3 we obtain

$$
\frac{d\left\langle m^{2}\right\rangle}{d t} \sim t^{2 \alpha_{c}(2-\tau)-1}+\frac{2 p^{2}}{(1+p)} .
$$

If we assume that the first term is the dominant term, then we reach a contradiction for $\tau$ (namely, $\tau=1$ ). The other alternative is to that $\left\langle m^{2}\right\rangle \sim t$, implying that $\alpha\left(3-\tau_{c}\right)=1$ or

$$
\alpha=\frac{2}{3} .
$$

Thus, in the mean field limit, the scaling function takes on the form

$$
P(m, \tilde{q}, t) \sim \frac{1}{m^{3 / 2}} Y\left(m \tilde{q}^{2}, \frac{m}{t^{2 / 3}}\right), \quad \mu=1 .
$$

$$
\text { 3. } \mu>0
$$

We will follow the same procedure as for the $\mu=0$ and the $\mu=1$ cases. However, for arbitrary $\mu$, we are no longer able to determine neither the critical value $q_{c}$ 
nor the exponent $\phi$. Consider the equations arising from choosing $n=2$ and $n=3$ in Eq. (B33) in the steady state:

$$
\begin{aligned}
\left\langle m^{1-\mu}\right\rangle & =p-q-\frac{q}{\langle m\rangle}, \\
\left\langle m^{2-\mu}\right\rangle & =\frac{q\left\langle m^{2}\right\rangle}{\langle m\rangle^{2}}-(p+q) .
\end{aligned}
$$

To satisfy Eq. (B30), we require that $\langle m\rangle$ diverges at the critical point. Substituting the scaling form, we obtain

$$
\phi\left(3-\mu-\tau_{c}\right)=\phi\left(3-\tau_{c}\right)-2 \phi\left(2-\tau_{c}\right)
$$

implying that

$$
\tau_{c}=2-\frac{\mu}{2} .
$$

To calculate the exponent $\alpha$, all we require is that $\left\langle m^{1-\mu}\right\rangle$ is finite at the critical point and is equal to $p-q$. This follows from Eq. (B29). Now, if we stay at the transition point, we obtain that $d\left\langle m^{2}\right\rangle / d t=2 q$ implying that

$$
\left\langle m^{2}\right\rangle \sim t
$$

Therefore $\alpha\left(3-\tau_{c}\right)=1$ or

$$
\alpha=\frac{2}{2+\mu} .
$$

Thus, in the mean field limit, the scaling function takes on the form

$$
P(m, \tilde{q}, t) \sim \frac{1}{m^{2-\mu / 2}} Y\left(m \tilde{q}^{\phi}, \frac{m}{t^{2 /(2+\mu)}}\right), \quad 0<\mu<2 .
$$

\section{APPENDIX C: SOLUTION FOR $d=0$}

In zero dimensions, the problem becomes analytically tractable as diffusion no longer plays a role. Therefore, the exponents are independent of $\mu$. The master equation for the evolution of the mass distribution $P(m)$ is

$$
\begin{aligned}
& \frac{d P(m)}{d t}=-(p+q) P(m)+p P(m+1)+q P(m-1)(\mathrm{C} 1) \\
& \frac{d P(0)}{d t}=-q P(0)+p P(1) .
\end{aligned}
$$

The steady state solution is obtained by setting the time derivatives to zero. It is then straightforward to obtain

$$
P(m)=\frac{p-q}{p}\left(\frac{q}{p}\right)^{m}, \quad m \geq 0
$$

This solution is valid when $q<p$. For $q \geq q_{c}=p$, there is no nontrivial steady state solution. The typical mass diverges as $q$ approaches $q_{c}$ as $\left(q-q_{c}\right)^{-1}$; therefore

$$
\phi=1
$$

Also, the occupation probability $s=1$ when $q=q_{c}$. Since $s$ cannot increase beyond 1 , it remains stuck at 1 for all further values of $q$. When $\tilde{q}=q-q_{c}$ is positive, $d\langle m\rangle / d t=q-p s=\tilde{q}$. This means that $\theta=1$.

The exponents $\tau_{c}$ and $\alpha$ may be obtained by solving the problem at $q=q_{c}$. In this case if one were to identify $m$ as the coordinate of a random walker, then the problem reduces to a problem of a random walker with a reflecting barrier at the origin. This problem is easily solved 22] and in the limit of large time,

$$
P(m, t) \approx \frac{1}{\sqrt{\pi q t}} \exp \left(\frac{-m^{2}}{4 q t}\right), t \gg 1
$$

The exponents $\tau_{c}$ and $\alpha$ may be read off from Eq. (C5) to be

$$
\begin{aligned}
\tau_{c} & =1, \\
\alpha & =\frac{1}{2},
\end{aligned}
$$

in zero dimensions.

When $q>q_{c}$, the problem reduces to the problem of a random walker with a drift and a reflecting barrier at the origin. Again, this problem is easily solvable:

$$
P(m, t)=\frac{1}{m} \frac{m}{(q-p) t} \delta\left(\frac{m}{(q-p) t}-1\right), \quad m, t \gg 1 .
$$

Clearly, the exponent $\gamma=1$ (see Eq. (15) for definition).
[1] J. A. Venables, G. D. T. Spiller, and M. Hanbücken, Rep. Prog. Phys. 47, 399 (1984).

[2] A. E. Scheidegger, Bull. I.A.S.H. 12, 15 (1967).

[3] P. S. Dodds and D. H. Rothman, Phys. Rev. E 59, 4865 (1999).

[4] S. K. Friedlander, Smoke, Dust and Haze (Wiley Interscience, New York, 1977).

[5] W. H. White, J. Colloid Interface Sci. 87, 204 (1982).

[6] R. M. Ziff, J. Stat. Phys. 23, 241 (1980).
[7] S. N. Majumdar, S. Krishnamurthy, and M. Barma, Phys. Rev. Lett. 81, 3691 (1998).

[8] S. N. Majumdar, S. Krishnamurthy, and M. Barma, Phys. Rev. E 61, 6337 (2000).

[9] U. Alon, M. R. Evans, H. Hinrichsen, and D. Mukamel, Phys. Rev. Lett. 762746 (1996); Phys. Rev. E 574997 (1998).

[10] H. Hinrichsen, R. Livi, D. Mukamel, and A. Politi, Phys. Rev. Lett. 79, 2710 (1997). 
[11] M. Takayasu and H. Takayasu in Nonequilibrium Statistical Mechanics in One Dimension ed. V. Privman (Cambridge Univ. Press, Cambridge, 1997).

[12] M. R. Swift, F. Colaiori, A. Flammini, A. Maritan, A. Giacometti and J. R. Banavar, Phys. Rev. Lett. 79, 3278 (1997).

[13] R. Rajesh and S. N. Majumdar, Phys. Rev. E 62, 3186 (2000).

[14] P. L. Krapivsky, J. F. F. Mendes, and S. Redner, Phys. Rev. B 59, 15950 (1999); Eur. Phys. J. B 4, 401 (1998).

[15] J. Camacho, Phys. Rev. E 63, 046112 (2001).

[16] C. Connaughton, R. Rajesh and O. Zaboronski, cond-mat/0310063

[17] H. Takayasu, Phys. Rev. Lett. 63, 2563 (1989).

[18] T. Nagatani, J. Phys. A 26, L489 (1993).

[19] S. N. Majumdar and C. Sire, Phys. Rev. Lett. 71, 3729 (1993).

[20] R. Rajesh and S. N. Majumdar, Phys. Rev. E 63, 036114 (2001).

[21] R. Rajesh, D. Das, B. Chakraborty, and M. Barma, Phys. Rev. E 66, 056104 (2002).

[22] W. Feller, An introduction to probability theory and its applications (Wiley, New York, 1970). 